\title{
Optimal duration and predictors of diagnostic utility of patient-activated ambulatory ECG monitoring
}

\author{
Eugene S J Tan, Swee-Chong Seow, Pipin Kojodjojo, Devinder Singh, Wee Tiong Yeo, \\ Toon Wei Lim
}

- Additional material is published online only. To view please visit the journal online (http://dx.doi.org/10.1136/ heartasia-2018-011061).

Department of Cardiology, National University Heart Centre Singapore, Singapore, Singapore

\section{Correspondence to} Dr Eugene S J Tan, National University Heart Centre Singapore, Singapore 119074, Singapore; eugene_sj_tan@ nuhs.edu.sg

Received 4 May 2018 Revised 8 August 2018 Accepted 7 October 2018

Check for updates

(C) Author(s) (or their employer(s)) 2018. No commercial re-use. See rights and permissions. Published by BMJ.

To cite: Tan ESJ, Seow S-C, Kojodjojo P, et al. Heart

Asia 2018;10:e011061.

doi:10.1136/

heartasia-2018-011061

\section{ABSTRACT}

Objective We studied the optimal duration of ambulatory event monitors for symptomatic patients and the predictors of detected events.

Methods Patients with palpitations or dizziness received a patient-activated handheld event monitor which records 30 s single-lead ECG strips. Patients were monitored in an ambulatory setting for a range of 1-4 weeks and ECG strips interpreted by five independent electrophysiologists. Event pick-up rates and clinical covariates were analysed.

Results Of 335 consecutive adults (age $50 \pm 16$ years, $58 \%$ female) with palpitations ( $94 \%$ ) and dizziness (25\%) monitored, 286 patients (85\%) reported events, and clinically significant events were detected in 86 (26\%) patients. Of these 86 patients, $26 \%$ had $\geq 2$ significant events, and $73 \%$ had events detected in the first 3 days. No significant events were detected after 12 days. The most common ECG abnormalities detected were premature ventricular ectopy (38\%), premature atrial ectopy $(37 \%)$ and atrial fibrillation (AF)/atrial flutter (34\%). A history of AF (adjusted OR (AOR) $4.2,95 \% \mathrm{Cl} 1.1$ to 15.8 ), previous arrhythmia (AOR 2.8, 95\% Cl 2.3 to 5.9) and previous abnormal ambulatory monitoring (AOR 3.4, 95\% Cl 1.0 to 9.4) were associated with detection of clinically significant events. Patients older than 50 years were $82 \%$ more likely to have a clinically significant event (OR 1.8, 95\% Cl 1.3 to 3.6).

Conclusion Patient-activated ambulatory event monitoring for 7 days may be sufficient in the diagnosis of symptomatic patients as significant events first detected beyond 10 days were rare. Patients with a history of AF, arrhythmia or previous abnormal ambulatory monitoring may require even shorter monitoring periods.

\section{INTRODUCTION}

Palpitations and dizziness are common presentations in the outpatient setting and emergency department (ED). The estimated prevalence of clinical visits for palpitations ranges from $8.3 \%{ }^{1}$ to 5.8 per $1000 \mathrm{ED}$ visits. ${ }^{2}$ The transient nature of these symptoms and ECG changes mean that a large proportion of arrhythmias remain undetected when patients present to an ECG-capable facility. In patients with infrequent symptoms, 24-hour to 48-hour Holter monitoring has a poorer diagnostic yield compared with other forms of prolonged monitoring. ${ }^{34}$

The utility of patient-activated event recorders in the detection of arrhythmic causes of infrequent palpitations and giddiness is widely recognised, ${ }^{56}$

\section{Key messages}

What is already known about this subject?

- In patients with infrequent symptoms of palpitations and dizziness, 24-hour Holter monitoring is of low diagnostic yield. The use of patient-activated event monitoring allows symptom correlation with detected arrhythmias that are clinically significant. However, the optimal duration of monitoring remains unclear and varies between studies.

What does this study add?

- This study demonstrates that patient-activated ambulatory ECG monitoring was able to pick up an arrhythmic event in most symptomatic patients within the first 3 days, with all clinically significant events detected within 14 days of monitoring. Monitoring beyond 2 weeks might not increase diagnostic yield significantly in symptomatic patients.

How might this impact on clinical practice?

- Patient-activated ambulatory ECG monitoring for 7 days, up to a maximum of 14 days, may be sufficient in the diagnostic evaluation of patients with palpitations or giddiness. In the subset of patients with pre-existing atrial fibrillation, arrhythmias or previous abnormal ambulatory monitoring, an even shorter duration of monitoring may suffice when assessing for recurrence of symptomatic arrhythmias.

with leadless devices gaining popularity due to their ease of use and improved patient compliance. ${ }^{67}$ The use of ambulatory patient-operated ECG device without supervision by healthcare personnel for arrhythmia detection had been previously validated. ${ }^{6}$ The clinical significance of arrhythmia detection lies in its therapeutic implications, with atrial fibrillation (AF), atrial flutter, supraventricular tachycardia, ventricular tachycardia (VT), sinus pause and atrioventricular block all necessitating different treatment strategies.

Monitoring periods of $\leq 14$ days $^{7}$ as opposed to a minimum of 2 weeks ${ }^{8}$ have been suggested, but the optimal duration remains unclear. In this study, we seek to determine the optimal duration of patient-activated ambulatory ECG monitoring and the predictors of detecting a clinically significant arrhythmic event. 

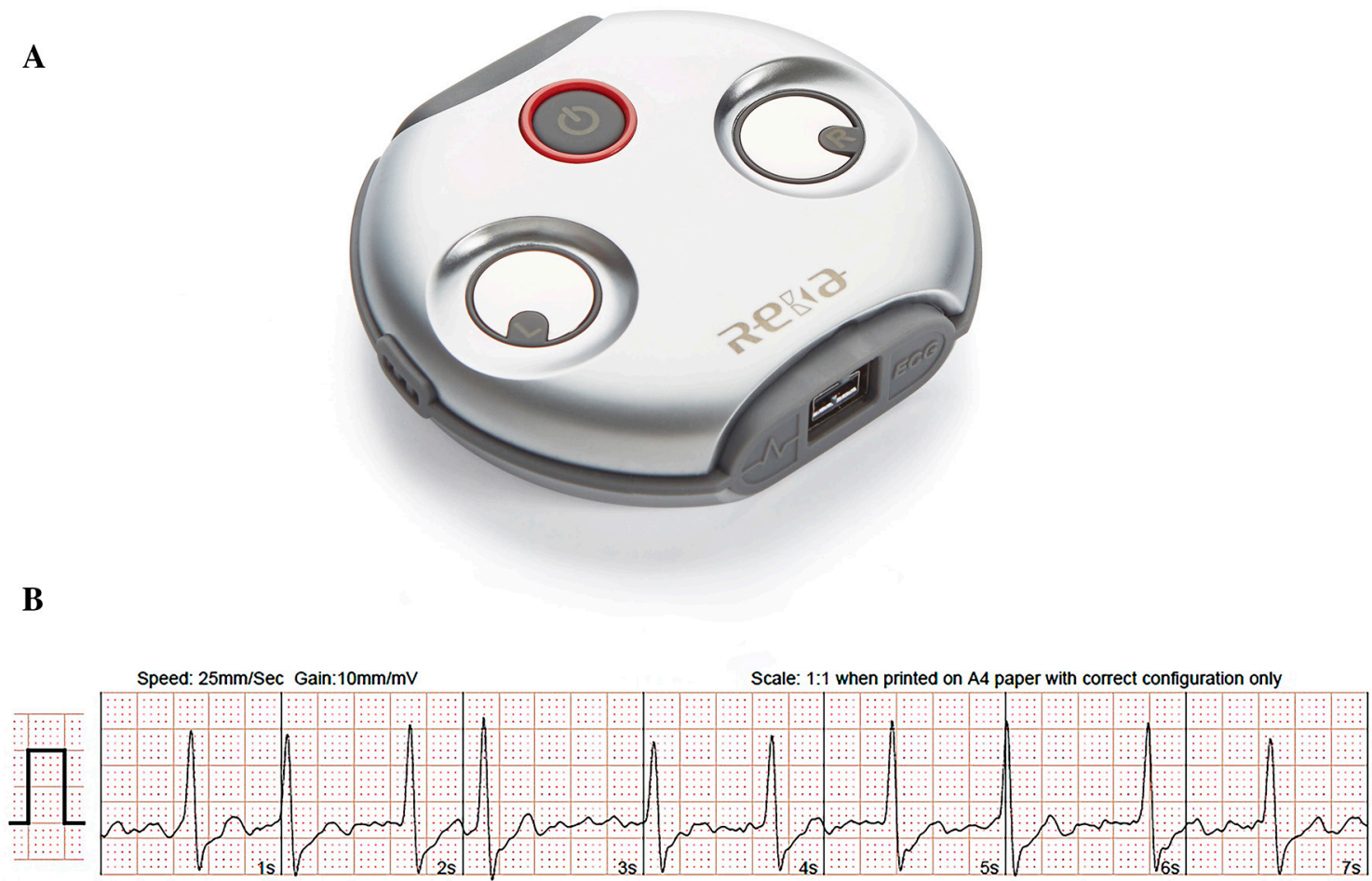

Figure 1 (A) Photograph of the handheld patient-activated ambulatory ECG monitoring device. (B) Example of patient-activated single-lead ECG strip recording during sensation of palpitations.

\section{METHODS}

\section{Study population}

Participants included in this study were patients who had presented to cardiology clinic from 2013 to 2016, with symptoms of palpitations and dizziness occurring less than once per week. As part of their diagnostic workup, patients were monitored on the patient-activated handheld ECG monitoring device (REKA E100, REKA, Singapore). All patients monitored with the handheld ECG monitoring device were included only if they were above 21 years of age and were symptomatic at presentation. Baseline demographics, clinical history and resting 12-lead ECGs were obtained at the index clinic visit or inpatient hospital admission.

ECG left ventricular hypertrophy was derived by either the Sokolow-Lyon voltage criteria ${ }^{9}$ or Cornell voltage criteria. ${ }^{10}$ ECG parameters of interest include PR interval, QRS duration, corrected QT interval by Bazett's formula ${ }^{11}$ cardiac axis and bundle branch block morphology.

\section{Patient-activated device monitoring}

As part of the standard workup and evaluation for their symptoms, all patients were monitored with a handheld ECG monitoring device for a period of 1-4 weeks in an ambulatory setting. The duration of monitoring was at the ordering physician's discretion. Patients were instructed to record a $30 \mathrm{~s}$ single-lead ECG strip either by placing their thumbs on the device's integrated platinum electrodes or through wired ECG electrodes applied to their chest (figure 1A). They were also asked to record a symptom diary at the same time.

At the end of the monitoring period, device-recorded ECG rhythm strips were interpreted by five independent electrophysiologists (figure 1B). Diagnostic events were defined as those with interpretable ECG rhythm strips at the time of patient symptom. Clinically significant events were defined as arrhythmias detected that may alter a patient's clinical management and these included
$\mathrm{AF} /$ atrial flutter, atrial tachycardia (AT), supraventricular tachycardia (SVT), premature atrial ectopic beats (PAC), premature ventricular ectopic beats (PVC), atrioventricular nodal block or sinus arrest and VT. Of note, premature atrial and ventricular ectopic beats were included as clinically significant due to their ability to cause symptoms and distress to patients. Sinus arrhythmia and sinus tachycardia were considered physiological and for which there is no specific therapy and hence were not considered to be clinically significant. All ECG rhythm strips were correlated with the patient's reported symptoms.

\section{Statistical analysis}

Baseline characteristics were reported as percentages (\%) for categorical variables and mean $\pm S D$ for continuous variables. Differences in baseline characteristics were compared with bivariate analyses with $\chi^{2}$ test or independent samples t-test where appropriate. Univariate analysis by binary logistic regression was then performed to determine clinical predictors of detection of a clinically significant event. Variables with a p value of $<0.1$ were then included in a multivariate analysis to determine its association with clinically significant events.

Further analyses were then performed in patient subgroups to compare baseline differences by either $\chi^{2}$ test (categorical) or independent samples t-test (continuous). These included the detection of events within 7 days and those with a history of AF or arrhythmia or previous abnormal ambulatory ECG monitoring. A p value of $<0.05$ was considered significant.

Cumulative probability of diagnostic, clinically significant events and atrial arrhythmias (AF/atrial flutter, SVT and AT) were plotted with the Kaplan-Meier curves (figures 2-4 respectively). Allocation bias was accounted for by multivariate analysis of patient factors in the association of predictors with clinically significant events, while consecutive patients were included in this study to reduce selection bias. Variables included in the multivariable logistic regression model in the predictors of 


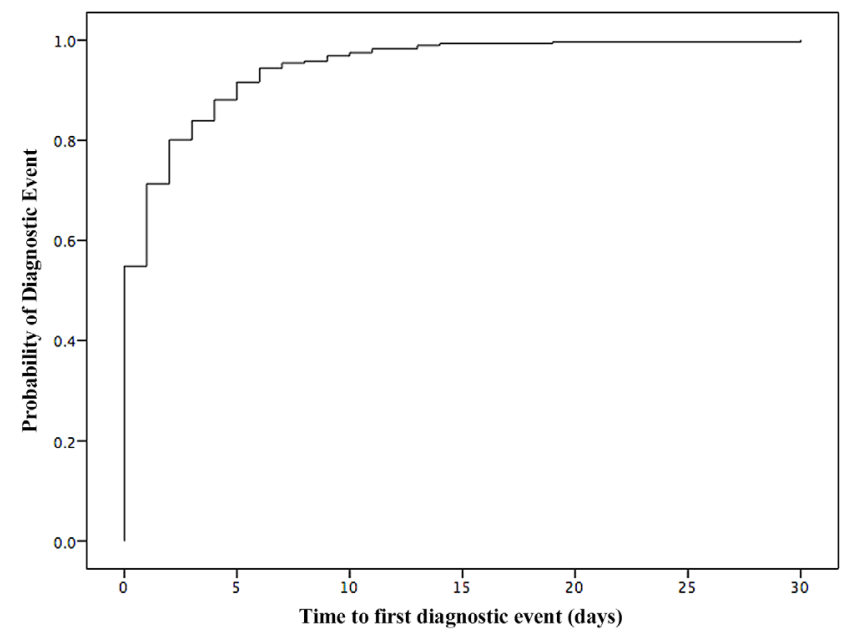

Figure 2 Kaplan-Meier curve of cumulative incidence of diagnostic events detected over the duration of monitoring.

significant events were those with $\mathrm{p}<0.2$ in univariable analysis. Backward elimination was performed to attain the final multivariate model. All patients were accounted for, and there were no patients lost to follow-up or missing data for analysis.

\section{RESULTS}

A total of 335 consecutive adults (age $50 \pm 16$ years, $58 \%$ female) with palpitations (94\%) and dizziness (25\%) were monitored in the study. A total of 310 (93\%) patients were monitored for 1-2 weeks, while $25(7 \%)$ were monitored for 3-4 weeks. While monitored, 286 patients (85\%) had diagnostic events, but only $86(26 \%)$ patients had clinically significant events detected. Baseline characteristics of the study population are shown in table 1. Among 286 patients with diagnostic events, 240 (72\% of total population) were reported within the first 3 days, 273 (81\% of total population) were reported at 1 week and 284 (85\% of total population) at 2 weeks. Only two patients had a diagnostic event detected beyond 2 weeks, but neither of which were significant events.

Of the 86 patients with detected events, 94\% were monitored for 1-2 weeks, while 6\% were monitored for 3-4 weeks.

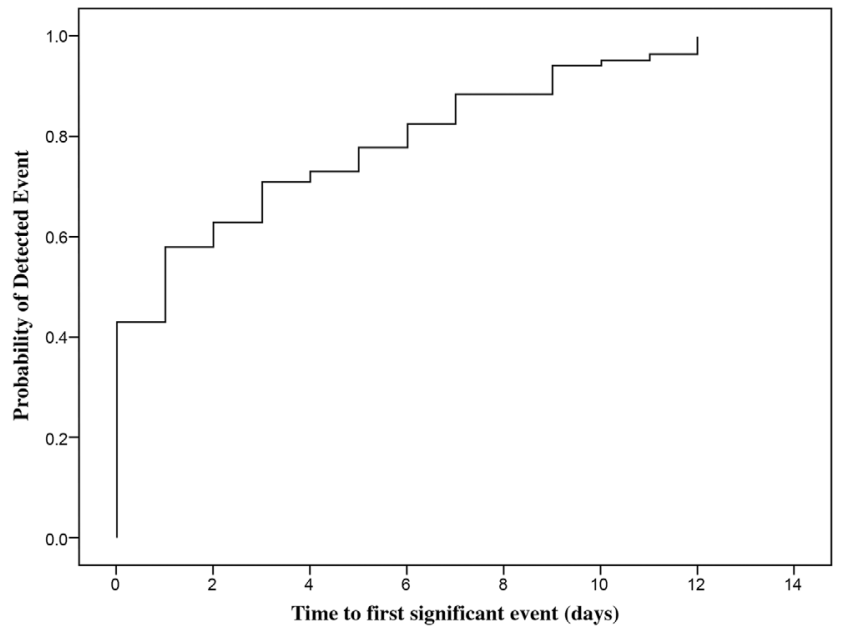

Figure 3 Kaplan-Meier curve of cumulative incidence of clinically significant events detected over the duration of monitoring.

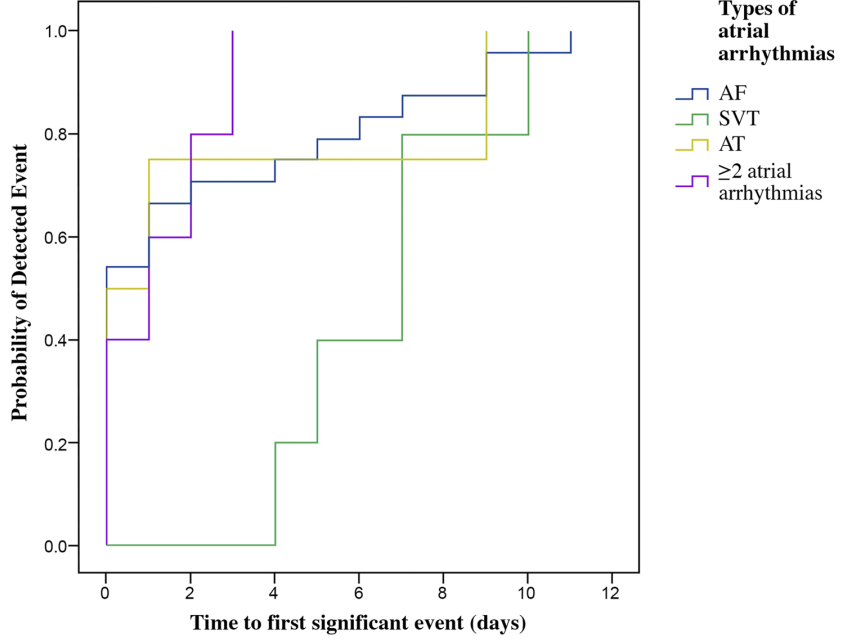

Figure 4 Kaplan-Meier curve of cumulative incidence of atrial arrhythmias detected over the duration of monitoring. $A F$, atrial fibrillation; AT, atrial tachycardia; SVT, supraventricular tachycardia.

Majority of patients with clinically significant events (73\%) were detected in the first 3 days, and a further $15 \%$ of patients had events detected between days 4-7. All patients had significant events detected within 14 days, and no significant events were detected after 14 days in those monitored for more than 2 weeks. Kaplan-Meier probability curves of diagnostic events and clinically significant events are shown in figures 2 and 3.The most common clinically significant event detected was premature ventricular ectopic beats (39\%), followed by premature atrial ectopic beats (38\%) and AF/atrial flutter (33\%) (table 2). Twenty-two patients had more than one type of arrhythmic event detected. Atrial arrhythmias including AF/atrial flutter, SVT and AT were likely to be detected within the first 3 days of monitoring (figure 4).

By univariate analysis, age (OR 1.02, 95\% CI 1.00 to 1.03 ), pre-existing AF (OR 6.57, 95\% CI 2.38 to 18.10), history of arrhythmias other than AF (OR $3.9695 \%$ CI 2.06 to 7.62), previous ambulatory monitoring (OR 1.78, 95\% CI 1.06 to 2.91), previous abnormal ambulatory monitoring (OR 5.04, 95\% CI 2.46 to 10.33 ) and beta-blockers (OR 2.14, 95\% CI 1.29 to 3.56 ) were associated with a clinically significant event. Patients older than 50 years were $82 \%$ more likely to have a clinically significant event (OR 1.82, 95\% CI 1.10 to 3.01) and every 10 -year increase in age was associated with almost $20 \%$ increased risk of a clinically significant event (OR 1.19, 95\% CI 1.02 to 1.38 ). By multivariate analysis, only those with a history of AF, arrhythmia and previous abnormal ambulatory ECG monitoring were associated with clinically significant events (table 3).

Comparing between those with a history of AF/arrhythmias or previous abnormal ambulatory monitoring and those without, significant differences were noted in age, detection of clinically significant events and time of onset. They were more likely to have AF or atrial flutter, AT, PACs and have more than one type of clinically significant event detected, and were more likely to be detected within the first 3 days of monitoring. This group of patients was also less likely to be in sinus rhythm, have longer PR interval, QRS duration and QTc interval (online supplementary table 1). There were no significant differences in characteristics for patients with events detected within 7 days compared with more than 7 days. 
Table 1 Baseline characteristics of patients with and without clinically significant events of patient-activated ambulatory ECG monitoring

\begin{tabular}{|c|c|c|c|}
\hline & $\begin{array}{l}\text { Significant } \\
\text { event }\end{array}$ & $\begin{array}{l}\text { Non-significant } \\
\text { event }\end{array}$ & $P$ values \\
\hline $\mathrm{N}$ & 86 & 249 & \\
\hline \multicolumn{4}{|l|}{ Demographics } \\
\hline Age, years & $54 \pm 17$ & $49 \pm 16$ & 0.03 \\
\hline Sex, female $n(\%)$ & $51(59)$ & $145(58)$ & 0.86 \\
\hline Ethnicity, Chinese n (\%) & $72(84)$ & $203(82)$ & 0.90 \\
\hline $\mathrm{BMI}, \mathrm{kg} / \mathrm{m}^{2}$ & $24.2 \pm 4.3$ & $24.1 \pm 5.0$ & 0.96 \\
\hline \multicolumn{4}{|l|}{ Clinical covariates } \\
\hline Palpitations n (\%) & $81(96)$ & $222(93)$ & 0.21 \\
\hline Dizziness n (\%) & $19(24)$ & $60(26)$ & 0.67 \\
\hline Family history of SCD n (\%) & $2(3)$ & $2(1)$ & 0.28 \\
\hline Known AF n (\%) & $12(14)$ & $6(2)$ & $<0.001$ \\
\hline Other arrhythmias $n(\%)$ & $22(27)$ & $21(8)$ & $<0.001$ \\
\hline Previous Holter n (\%) & $34(40)$ & $67(27)$ & 0.03 \\
\hline Previous abnormal Holter $\mathrm{n}(\%)$ & $21(24)$ & $15(6)$ & $<0.001$ \\
\hline Previous ablation n (\%) & $4(5)$ & $8(3)$ & 0.55 \\
\hline Echocardiographic EF n (\%) & $63 \pm 4$ & $63 \pm 6$ & 0.90 \\
\hline \multicolumn{4}{|l|}{ ECG parameters } \\
\hline $\mathrm{PR}, \mathrm{ms}$ & $167 \pm 42$ & $160 \pm 24$ & 0.045 \\
\hline QRS, ms & $92 \pm 17$ & $91 \pm 14$ & 0.58 \\
\hline QTc, ms & $432 \pm 29$ & $433 \pm 24$ & 0.76 \\
\hline Axis, degrees & $44 \pm 32$ & $42 \pm 40$ & 0.66 \\
\hline Sinus rhythm n (\%) & $79(98)$ & $237(98)$ & 0.86 \\
\hline LVH n (\%) & $3(4)$ & $20(9)$ & 0.14 \\
\hline BBB n (\%) & & & 0.28 \\
\hline Right & $5(6)$ & $6(3)$ & \\
\hline Left & $0(0)$ & $1(0.4)$ & \\
\hline \multicolumn{4}{|l|}{ Medications } \\
\hline Beta-blocker n (\%) & $38(44)$ & $68(27)$ & $<0.01$ \\
\hline Non-dihydropyridine CCB n (\%) & $3(4)$ & $7(3)$ & 0.76 \\
\hline Digoxin n (\%) & $1(1)$ & $0(0)$ & NA \\
\hline Other antiarrhythmics n (\%) & $2(2)$ & $2(1)$ & NA \\
\hline Duration of monitoring & & & 0.13 \\
\hline 1 Week n (\%) & $1(1)$ & $20(8)$ & \\
\hline 2 Weeks n (\%) & $80(93)$ & $210(84)$ & \\
\hline 3 Weeks n (\%) & $5(6)$ & $19(8)$ & \\
\hline 4 Weeks n (\%) & $0(0)$ & $1(0.4)$ & \\
\hline
\end{tabular}

Comparison of baseline characteristics of patients with and without detected clinically significant events.

$\mathrm{AF}$, atrial fibrillation; $\mathrm{NA}$, not available.

\section{DISCUSSION}

In this study, we showed that patient-activated ambulatory ECG monitoring was able to pick up an arrhythmic event in most symptomatic patients within the first 3 days, with all clinically significant events detected within 14 days of monitoring. A history of AF or other arrhythmias and previous abnormal ambulatory monitoring were predictors of detecting a clinically significant event.

\section{Diagnostic yield}

In our centre, patient-activated ECG monitoring yielded a clinical diagnosis in $85 \%$ of patients, with a diagnostic yield of $72 \%$ at 3 days, $81 \%$ at 1 week and $85 \%$ at 2 weeks. This was comparable with the large, multicentric SYNARR-Flash study which reported a diagnostic yield of $71.6 \%$ at 4 weeks in patients with unexplained palpitations. ${ }^{12}$ Clinically significant events were
Table 2 Incidence of clinically significant events and detection rates

\begin{tabular}{lc}
\hline & Number of events \\
\hline Type of event & $29(34)$ \\
\hline Atrial fibrillation/flutter (\%) & $8(9)$ \\
\hline SVT (\%) & $7(8)$ \\
\hline Atrial tachycardia (\%) & $32(37)$ \\
\hline PAC (\%) & $33(38)$ \\
\hline PVC (\%) & $1(1)$ \\
\hline AV block/sinus arrest (\%) & $1(1)$ \\
\hline Ectopic atrial rhythm (\%) & $22(26)$ \\
\hline$\geq 2$ types of events & $63(73)$ \\
\hline Time to first clinically significant event (number of patients=86) \\
\hline $0-3$ days (\%) & $13(15)$ \\
\hline $4-7$ days (\%) & $6(7)$ \\
\hline $8-10$ days (\%) & $4(5)$ \\
\hline $11-14$ days (\%) & $0(0)$ \\
\hline$\geq 15$ days (\%) & Incidence of clinically significant events and time to first detection. \\
SVT, supraventricular tachycardiaAV, atrioventricular; PAC, premature atrial ectopic beats; \\
\hline PVC, premature ventricular ectopic beats.
\end{tabular}

detected in $26 \%$ of patients, comparable with and even slightly higher than previous studies, where patient-triggered monitoring yielded a diagnosis in $16 \%-22 \%$, but lower than that in the SYNAAR-Flash study, where $45 \%$ of patients had arrhythmic events. ${ }^{51213}$ The different pickup rates observed are likely due to different definitions of 'significant events' and differences in study population characteristics. Premature atrial and ventricular ectopic beats were included in our definition of clinically significant arrhythmias due to their ability to cause physical and emotional distress in symptomatic patients. In a Swiss cohort of 1742 participants, up to $99 \%$ had at least one PAC detected on 24-hour Holter monitoring, ${ }^{14}$ while PVCs were detected in $1 \%-4 \%$ of the general population. ${ }^{15}$ Patients with a high PVC burden may develop cardiomyopathy if left untreated, ${ }^{16}$ while excessive PACs are also associated with stroke and AF. ${ }^{17}$ Detection of these premature ectopic beats is thus clinically important, especially if patients are highly symptomatic from them. Although the SYNARR-Flash study also included patients with ectopic beats, the difference in 'significant event' rates with our study is likely explained by the difference in inherent study population characteristics and inclusion of automatic recording modalities in the SYNARR-flash study. ${ }^{12}$

Table 3 Predictors of detection of clinically significant events

\begin{tabular}{|c|c|c|c|}
\hline Variables & Adjusted OR & $95 \% \mathrm{Cl}$ & $P$ values \\
\hline Age per 10 years & 1.01 & 0.84 to 1.21 & 0.96 \\
\hline Known AF/atrial flutter & 4.16 & 1.10 to 15.76 & 0.04 \\
\hline Previous arrhythmia & 2.75 & 1.29 to 5.90 & $<0.01$ \\
\hline $\begin{array}{l}\text { Previous ambulatory ECG } \\
\text { monitoring }\end{array}$ & 0.68 & 0.32 to 1.45 & 0.32 \\
\hline $\begin{array}{l}\text { Previous abnormal } \\
\text { ambulatory ECG } \\
\text { monitoring }\end{array}$ & 3.36 & 1.02 to 9.42 & 0.02 \\
\hline PR interval & 1 & 0.99 to 1.01 & 0.38 \\
\hline Beta blocker & 1.42 & 0.78 to 2.60 & 0.25 \\
\hline \multicolumn{4}{|c|}{$\begin{array}{l}\text { Multivariate analysis of clinical variables with detection of clinically significant events. } \\
\text { Comparison of baseline characteristics of patients with previous AF/atrial flutter or } \\
\text { arrhythmia or abnormal ambulatory ECG monitoring. } \\
\text { AF, atrial fibrillation; AT, atrial tachycardia; BMI, body mass index; PAC, premature } \\
\text { atrial ectopics beats; PVC, premature ventricular ectopics beats; SVT, supraventricular } \\
\text { tachycardia. }\end{array}$} \\
\hline
\end{tabular}

Tan ESJ, et al. Heart Asia 2018;10:e011061. doi:10.1136/heartasia-2018-011061 
Other studies have shown a higher yield of arrhythmic events with concurrent patient-activated and continuous monitoring. Turakhia et al showed a higher arrhythmic event pickup rate of $60 \%$ with the leadless long-term monitoring patch and symptom trigger button, ${ }^{7}$ while Balmelli et al reported a higher arrhythmia detection yield of $84 \%$ vs $16 \%$ in automatic recording and patient-triggered recording, respectively. ${ }^{13}$ In a randomised study of ambulatory monitoring, continuous mobile cardiac outpatient telemetry had a higher diagnostic yield of $88 \%$ compared with $75 \%$ in patient-activated external loop event monitor. ${ }^{18}$ These studies however included patients with syncope in whom continuous monitoring was more relevant as cardiac syncope tended to be sudden and patients might not react quickly enough to trigger their monitoring device before they were incapacitated. Our study was limited to symptomatic patients with only palpitations or giddiness, who were conscious throughout their symptoms and were able to activate the handheld monitoring device. Furthermore, although a continuously recording ambulatory monitoring system would offer a theoretically perfect yield in terms of capturing significant arrhythmic events, it comes with important limitations. The requirement to be physically attached to the patient throughout the monitoring period either as ECG leads or as an adhesive patch and the attendant discomfort of that result in poor patient compliance. Moreover, unless patients assiduously document all their symptoms, correlation with arrhythmias recorded may also be unreliable, and it may lead to overdiagnosis and unnecessary therapy. On the other hand, activation of handheld event monitors when patients experience symptoms and are conscious is more helpful in the clinical correlation of a detected clinically significant arrhythmic event as patients only record when they are indeed symptomatic.

Importantly, the absence of 'significant arrhythmias' in patients who activated their recorders while symptomatic may still be of clinical relevance. The exclusion of the presence of arrhythmias at the time of symptoms is helpful in the clinical management of symptomatic patients, who can be reassured of a benign aetiology of their symptoms. The high diagnostic yield of ambulatory ECG monitoring in this study, based on the likelihood of symptom recurrence within the monitoring period and availability of interpretable ECG tracings at the time of symptoms, makes it a highly useful tool in the evaluation of patients with unexplained but well-tolerated palpitations.

\section{Duration of ambulatory ECG monitoring}

The optimal duration of ambulatory ECG monitoring compared with the 24-hour Holter monitor remains unclear. The recent International Society for Holter and Noninvasive Electrocardiology and Heart Rhythm Society (ISHNE-HRS) expert consensus statement on ambulatory ECG monitoring and external cardiac monitoring/telemetry recommends the use of ambulatory ECG monitoring in patients with unexplained palpitations, with the choice of monitoring modality dependent on frequency of symptoms. ${ }^{19}$ At present, monitoring duration ranges from 1 week $^{9}$ to 1 month. ${ }^{50}$ Turakhia et al found that extended monitoring with the Zio Patch for $\leq 14$ days was feasible, with an incremental diagnostic yield beyond 48 hours, ${ }^{7}$ while Hoefman et al found that a minimum recording time of 2 weeks was necessary, with $79 \%$ of patients registering a relevant arrhythmia by the second week of monitoring. ${ }^{8}$ A 2 -week monitoring period was also recommended as the standard due to its cost-effectiveness and decreased diagnostic yield after 2 weeks. ${ }^{21}$ Balmelli et al showed that in patients monitored up to 7 days, $82 \%$ had a clinically relevant arrhythmia, although only $16 \%$ of these patients were patient-activated, with the rest detected automatically through continuous loop recording. ${ }^{13}$ This was similar to Zimetbaum et al's study, where $80 \%$ of patients had at least one diagnostic event within the first week, although only $28 \%$ were considered clinically relevant. ${ }^{21}$

In our study, we found that $73 \%$ of patients had a clinically relevant arrhythmia with symptom correlation within the first 3 days, and a further 15\% between days 4-7 of monitoring. The high detection rates were consistent with Zimetbaum et al's study ${ }^{21}$ and may have differed slightly due to our classification of PACs and PVCs as clinically relevant. These premature ectopic beats were included as patients were symptomatic from them and effected a change in management strategy as compared with patients with asymptomatic premature ectopic beats. In the group of patients who were monitored for more than 2 weeks, no significant events were detected after the second week. Moreover, a diminishing yield towards the end of the 2-week period suggests that monitoring for longer than that may not increase diagnostic yield significantly. Our findings also suggest that in patients usually monitored for 2 weeks, a shorter duration of 1 week may be sufficient as most events are picked up early in the monitoring period. In a select group of patients with pre-existing AF or arrhythmia or previous abnormal ambulatory monitoring, an even shorter duration of less than a week may be sufficient when assessing for recurrence. Whether there are patients who require prolonged monitoring remains uncertain as only $7 \%$ of patients were monitored for 3-4 weeks, and it was not possible to determine what patient characteristics were associated with later detection of arrhythmias. It is worth noting though that $80 \%$ of our patients who were monitored beyond 2 weeks had an event detected within the first 3 days which is consistent with the rest of the cohort who were monitored for 2 weeks or less.

In line with the ISHNE-HRS expert consensus statement, extended ambulatory ECG monitoring beyond 48 hours is recommended in patients with infrequent symptoms. ${ }^{19}$ However, a shorter duration of 7 days, up to 2 weeks, may be sufficient, with even shorter monitoring required in a select group of patients.

\section{Predictors of clinically significant events}

In the present study, patients with a known history of AF or arrhythmias, or a previous abnormal ambulatory ECG monitoring were more likely to have a clinically significant event detected in the evaluation of symptom recurrence.

Although the association of age with a clinically significant event was attenuated after multivariate adjustment, a trend towards detection of clinically significant events was noted in patients above 50 years of age, and for every 10-year increase in age. The association of age with arrhythmias from this study is well supported by previous studies which showed a higher prevalence of arrhythmias in elderly men on ECG. ${ }^{1}$ The Cardiovascular Health Study also showed an increase in prevalence of supraventricular and ventricular arrhythmias, and ectopic beats with age in both men and women, with an excess prevalence noted in men. ${ }^{22}$ These sex-differences were, however, not noted in our study.

In patients with a previous history of $\mathrm{AF}$, arrhythmia or abnormal ambulatory ECG monitoring, we found significant differences in age and detection time. Patients were older and were more likely to have a clinically significant event detected within the first 3 days of monitoring $86 \%$ vs $59 \%$. The higher age in patients with a history of arrhythmia or abnormal ambulatory monitoring further supports the association of age with clinically significant events. The high detection rates $97 \%$ vs $81 \%$ within the first week of monitoring suggests patients with 
a history of $\mathrm{AF} /$ arrhythmias or previous abnormal ambulatory monitoring may not require prolonged ambulatory monitoring for arrhythmia recurrence as events tend to be captured early during monitoring. In this group of patients, monitoring of less than a week's duration may even be sufficient.

When analysed by detection of events at $\leq 7$ days vs $>7$ days, we did not find a significant difference in age and sex, suggesting that duration of monitoring may not be age and sex specific.

\section{Limitations}

This study was limited to patient-activated triggered events and does not include continuous automatic loop recording. Clinically relevant arrhythmias with potential implications on treatment strategies in asymptomatic patients may remain undetected.

The assessment of palpitation characteristics including frequency, duration and regularity are not consistently recorded during routine clinic visits. These may have an impact on arrhythmia detection and duration of recording and may improve future clinical decisions on ambulatory ECG monitoring. Furthermore, the definition of 'significant arrhythmias' is arbitrary and dependent on the specific population under study which may have accounted for the difference in diagnostic yield in various studies.

Extension of the findings from this study to patients who require prolonged monitoring require further study due to the small number of patients who were monitored for 3-4 weeks.

\section{CONCLUSION}

Patient-activated ambulatory ECG monitoring for 7 days, up to a maximum of 14 days, may be sufficient in the diagnostic evaluation of patients with palpitations or giddiness. In the subset of patients with pre-existing AF, arrhythmias or previous abnormal ambulatory monitoring, an even shorter duration of monitoring may suffice when assessing for recurrence of symptomatic arrhythmias.

Acknowledgements The authors thank the technicians, nurses and staff at the
National University Heart Centre, Singapore, for their technical and clinical support.

Contributors All the coauthors listed in this manuscript fulfil criteria of authorship. ESJT contributed to the planning, data analysis, interpretation of data, reporting and writing of the manuscript. SCS, PK, DS and WTY contributed to the conduction of the study, interpretation of data and review of the manuscript. TWL contributed to the planning, conduction of study, interpretation of data and review of the manuscript.

Funding The authors have not declared a specific grant for this research from any funding agency in the public, commercial or not-for-profit sectors.

Competing interests None declared.

Patient consent Not required.

Ethics approval Ethics approval was obtained from the National Healthcare Group Domain Specific Review Board (Reference number 2015/00689).

Provenance and peer review Not commissioned; externally peer reviewed.

\section{REFERENCES}

1 Lok NS, Lau CP. Prevalence of palpitations, cardiac arrhythmias and their associated risk factors in ambulant elderly. Int J Cardiol 1996;54:231-6.

2 Probst MA, Mower WR, Kanzaria HK, et al. Analysis of emergency department visits for palpitations (from the National Hospital Ambulatory Medical Care Survey). Am J Cardiol 2014;113:1685-90.

3 Sivakumaran S, Krahn AD, Klein GJ, et al. A prospective randomized comparison of loop recorders versus Holter monitors in patients with syncope or presyncope. Am J Med 2003:115:1-5.

4 Barrett PM, Komatireddy R, Haaser S, et al. Comparison of 24-hour Holter monitoring with 14-day novel adhesive patch electrocardiographic monitoring. Am J Med 2014;127:95.e11-95.e17.

5 Hoefman E, van Weert HC, Reitsma JB, et al. Diagnostic yield of patient-activated loop recorders for detecting heart rhythm abnormalities in general practice: a randomised clinical trial. Fam Pract 2005;22:478-84.

6 Kaleschke G, Hoffmann B, Drewitz I, et al. Prospective, multicentre validation of a simple, patient-operated electrocardiographic system for the detection of arrhythmias and electrocardiographic changes. Europace 2009;11:1362-8.

7 Turakhia MP, Hoang DD, Zimetbaum P, et al. Diagnostic utility of a novel leadless arrhythmia monitoring device. Am J Cardio/ 2013;112:520-4.

8 Hoefman E, van Weert HC, Boer KR, et al. Optimal duration of event recording for diagnosis of arrhythmias in patients with palpitations and light-headedness in the general practice. Fam Pract 2007;24:11-13.

9 Sokolow M, Lyon TP. The ventricular complex in left ventricular hypertrophy as obtained by unipolar precordial and limb leads. Am Heart J 1949;37:161-86.

10 Okin PM, Roman MJ, Devereux RB, et al. Electrocardiographic identification of increased left ventricular mass by simple voltage-duration products. J Am Coll Cardiol 1995;25:417-23.

11 Bazett H. An analysis of time relations of the electrocardiogram. Heart 1920;7:353-70.

12 Locati ET, Moya A, Oliveira M, et al. External prolonged electrocardiogram monitoring in unexplained syncope and palpitations: results of the SYNARR-Flash study. Europace 2016:18:1265-72.

13 Balmelli N, Naegeli B, Bertel O. Diagnostic yield of automatic and patient-triggered ambulatory cardiac event recording in the evaluation of patients with palpitations, dizziness, or syncope. Clin Cardiol 2003;26:173-6.

14 Conen D, Adam M, Roche F, et al. Premature atrial contractions in the general population: frequency and risk factors. Circulation 2012;126:2302-8.

15 Kennedy HL, Whitlock JA, Sprague MK, et al. Long-term follow-up of asymptomatic healthy subjects with frequent and complex ventricular ectopy. N Engl J Med 1985;312:193-7.

16 Lee GK, Klarich KW, Grogan M, et al. Premature ventricular contraction-induced cardiomyopathy: a treatable condition. Circ Arrhythm Electrophysiol 2012;5:229-36.

17 Binici Z, Intzilakis T, Nielsen OW, et al. Excessive supraventricular ectopic activity and increased risk of atrial fibrillation and stroke. Circulation 2010;121:1904-11.

18 Rothman SA, Laughlin JC, Seltzer J, et al. The diagnosis of cardiac arrhythmias: a prospective multi-center randomized study comparing mobile cardiac outpatient telemetry versus standard loop event monitoring. J Cardiovasc Electrophysiol 2007;18:241-7.

19 Steinberg JS, Varma N, Cygankiewicz I. ISHNE-HRS expert consensus statement on ambulatory ECG and external cardiac monitoring/telemetry. Heart Rhythm 2017;2017:e55-96.

20 Locati ET, Vecchi AM, Vargiu S, et al. Role of extended external loop recorders for the diagnosis of unexplained syncope, pre-syncope, and sustained palpitations. Europace 2014;16:914-22.

21 Zimetbaum PJ, Kim KY, Josephson ME, et al. Diagnostic yield and optimal duration of continuous-loop event monitoring for the diagnosis of palpitations. A costeffectiveness analysis. Ann Intern Med 1998;128:890-5.

22 Manolio TA, Furberg CD, Rautaharju PM, et al. Cardiac arrhythmias on 24-h ambulatory electrocardiography in older women and men: the Cardiovascular Health Study. J Am Coll Cardiol 1994;23:916-25. 